Horizons philosophiques

\title{
Esthétique littéraire et informatique aléatoire
}

\section{François Raymond}

Volume 3, numéro 2, printemps 1993

Paysages esthétiques

URI : https://id.erudit.org/iderudit/800923ar

DOI : https://doi.org/10.7202/800923ar

Aller au sommaire du numéro

\section{Éditeur(s)}

Collège Édouard-Montpetit

\section{ISSN}

1181-9227 (imprimé)

1920-2954 (numérique)

Découvrir la revue

Citer cet article

Raymond, F. (1993). Esthétique littéraire et informatique aléatoire. Horizons philosophiques, 3(2), 77-88. https://doi.org/10.7202/800923ar d'utilisation que vous pouvez consulter en ligne.

https://apropos.erudit.org/fr/usagers/politique-dutilisation/ 


\section{Esthétique littéraire et informatique aléatoire}

Il semble qu'il soit bon de parler de peu de choses à la fois. Aussi m'en tiendrai-je à la description d'une machine informatique qui crée un grand nombre de phrases au hasard et à la présentation de quelques commentaires sur ses effets esthétiques et quelques usages possibles.

Les stylos, les plumes, les crayons, les dactylographes et les logiciels de traitement de texte servent, entre autres, à ceux qui savent déjà quoi écrire ou qui se fient à eux-mêmes pour construire des phrases, ou pour en citer. Mais pour quelqu'un qui a besoin d'un énoncé dont il ne connaît pas l'origine, dont il ne veut même pas connaître l'origine, d'une suite de phrases qui se répètent le moins possible, pour ceux qui, par stratégie, ont besoin d'énoncés étranges et très différents les uns des autres, pour le dramaturge qui veut simuler une conversation ou se donner un point de départ ou d'arrivée, pour le poète à la page blanche, pour l'explorateur du hasard des mots, pour le curieux des conjonctions disparates, cadavres exquis et autres ornithorynques, pour celui qui tente une nouvelle donne avec son lexique préféré, etc., le générateur d'énoncés aléatoires est là qui n'attend que son bon vouloir.

Une telle suite de coups de dés a cela de particulier qu'elle peutêtre retravaillée à qui mieux mieux, sans que qui que ce soit ne s'en soucie. Elle n'a ni morale, ni inconscient, ni conscience : pur produit de matière verbale brute ou raffinée. Selon les points de vue, la programmation, l'orientation

lexicale, l'usage, la rature, la suggestivité induite, la capacité de supporter l'absurde dans le langage et celle d'imaginer à partir d'une donnée cette longue séquence de phrases se trace et se retrace dans un chatoiement de paroles.

Un ordinateur adéquatement programmé produit une matière souple et très variée. Et la sensibilité qu'elle développe chez le lecteur tient son effet de distribution aléatoire, sorte de 
feuilleté de propos décousus qui tirent chacun dans leur direction et se servent mutuellement de contextes disparates. Lus à plusieurs, ces textes produisent un effet hilarant certain. Mais aussi, à mesure que les phrases s'écrivent à l'écran, on sent le martellement d'une parole d'indifférence qui danse sur les noms de l'histoire ou les anonymes Pierre Jean Jacques.

Il y a quatre niveaux d'écriture informatique : 1) celui de la machine avec l'inscription d'une architecture dans le microprocesseur; 2) celui de la constitution du langage de programmation qui agit sur le premier; 3) celui du logiciel écrit dans un langage de programmation; 4) celui, enfin, d'un opérateur qui utilisera ce dernier pour écrire des suites de phrases. Plus un procédé se répète plus il s'épuise. Sinon en fait, du moins dans la sensibilité qui le reçoit. Par fatigue, l'esprit se lasse. Aussi est-il pertinent de produire un procédé, si tant est qu'il faille en produire un, ouvert et qui se répète très peu souvent. Riches et explorables à souhait, les générateurs informatiques d'énoncés aléatoires ont cette qualité de ne pas se répéter. C'est leur qualité exploratoire et créatrice. Ils simulent la variation spontanée d'un esprit en pleine ébullition, la jonglerie avec la matière de la mémoire. À l'horizon persiste une vaste plage qui a la beauté du hasard et la causticité sobre de l'indifférence.

Donc, des programmes fonctionnent très bien sur des ordinateurs personnels. Leur vocabulaire, sans y être limité, est actuellement d'environ 9000 mots, à quoi s'ajoutent quelques centaines de structures de phrases. La machine fouille au hasard dans une banque de formes syntaxiques que s'est données l'utilisateur, en saisit une et se metà la remplir de mots au hasard. On peut faire varier l'aléatoire par degrés selon que certaines formes syntaxiques sont fixées ou livrées au même traitement. Par exemple, grâce au conjugueur de verbes, on choisira entre tous les temps d'un verbe ou seulement l'imparfait du subjonctif. 
En somme on peut ajouter, retrancher ou composer l'agencement syntaxique que l'on veut et y adapter n'importe quel vocabulaire. Et la machine écrit des phrases dont l'état se répète plus ou moins selon l'extension de la banque de structures syntaxiques et de lexiques qu'on y a liés. On peut fixer la récurrence d'un mot de sorte que les phrases générées tournent continuellement comme autour d'un axe. De même, une structure peut aussi être déterminée pour des contenus variables.

Qu'est-ce qu'on simule? Les citations incohérentes d'un inconnu, une série d'énoncés sur des sujets quelconques, la parole d'un délirant prolixe, ou les premiers balbutiements d'une machine cristallozoaire selon la prédiction de Samuel Butler dans Erewhon. Quand on lui donne toute liberté, la machine ne se répète pratiquement pas. En théorie elle devra se répéter parce que le nombre d'éléments permutés, bien qu'assez grand, n'est pas infini. Mais en pratique, c'est un chaos verbal, ligne après ligne.

Le programme nommé Prisme, dont on lira les productions à la fin de ce texte, est écrit en Turbo Prolog. II peut écrire beaucoup plus de phrases que j'en puis lire durant mon entière existence et, cela, en relativement peu de temps. D'où la belle absurdité d'une telle profusion de phrases sans fond, très souvent ineptes, quelquefois seulement pertinentes à un certain objet, mais évidemment toujours hors contexte.

La lecture est alors à l'image de la promenade sur une plage, à chercher des galets bien formés ou de formes et de tons singuliers. Quelqu'un qui sait ce qu'il cherche y trouvera peut-être ce qu'il veut? N'est-ce pas ça, après tout, la lecture! Non, les machines informatiques ne pensent pas, elles agencent les éléments qu'on leur fournit d'une façon formelle (c'està-dire exprimable en termes d'algorithme). Ces écrits donnent à penser bien des choses, comme dirait Kant, et en bric à brac. Symbole moderne, énigme à l'état brut, c'est le nouveau sublime. Ce sont des phrases qui ont l'apparence de la pensée. 
Comme il y a une esthétique de la répétition de formes semblables, il y en a une de la variété hétéroclite. Les surréalistes avaient une sensibilité toute proche avec les «cadavres exquis", mais la grande différence c'est que leurs productions tentaient de sonder l'inconscient, d'aller chercher ailleurs avec des formes disruptives, bref, d'exprimer. Tandis qu'ici il n'y a pas d'expression, pas d'intention, pas d'inconscient, pas de morale. Tout ça, au contraire, passe entièrement dans l'oeil du lecteur. Par ci, par là, celui-ci voit son goût choqué, ses principes baffoués, ses plus obscures intentions étalées crues. Ces choses qu'il porte en lui et qu'il projette autour baignant dans une profusion éclatée.

L'intéressant dans la lecture de ce genre d'écrit, c'est qu'à chaque phrase le lecteur refait tout un contexte pour que ce qu'il lit ait un sens. Avec certaines phrases, le sens surgit spontanément, parce qu'elles disent quelque chose de clair et reconnu comme «la lune est bleue" ou "c'est la dialectique locale et non pas un multiple noble qui particularise". Mais le plus souvent, à longueur de page, les propos incongrus et absurdes à première vue s'enfilent à la queue leu leu.

Cependant, il apparaît très vite que toute l'absurdité et l'incongruité ne sont pas entièrement du côté de la machine puisque le jugement ne vient que du lecteur et de sa capacité, plus ou moins grande, d'imaginer un contexte pertinent. Autrement dit, le même énoncé sera ineptie pour l'un, trouvaille pour l'autre. Et pour cette raison on peut nommer ce genre de production : littérature à construction contextuelle ou à contexte obligé. Elle est indifférente, innocente et insensible. Dans cet espace d'écriture, la langue s'émiette d'elle-même et la signification est à la charge de l'imagination, de l'humour, de l'opportunisme et du hasard. Quelques lecteurs tomberont, victimes du sérieux du sens et de l'idéologie. Tous ne mourront pas.

Personne n'écrit et sans cesse la langue se replie et se déplie, sous nos yeux, souvent sibylline, elle déploie la nouvelle énigme. 
Nous connaissons déjà un objet technique analogue qui produisit, à son apparition, des effets disrupteurs dans les métiers de la représentation. La photographie, au siècle passé, a profondément bouleversé alors le travail des professionnels occupés à la reproduction visuelle des choses du monde. Des corps de métier au complet sont disparus, un peu comme les forgerons à l'arrivée de l'automobile. La gravure, la reproduction d'art, le portrait ont été assez vite éliminés. Si la gravure existe encore aujourd'hui c'est en empruntant un autre chemin que celui de la reproduction réaliste du monde, que la photographie fait déjà si bien. Il en va de même pour la peinture. Tout ce qui en elle était reproduction ou doublure du réel tracées par la main subordonnée à l'oeil a été bouleversée par la précision, l'exactitude impitoyable de la photographie. Les métiers touchés ont tenté de négocier avec la nouvelle technique par l'exploration de nouveaux domaines inaccessibles à celle-ci. Par exemple, la peinture s'est mise à explorer la non-représentation et la déformation expressionniste, omniprésentes au $X X^{\ominus}$ siècle.

Notre analogie suppose que les générateurs informatiques sontà l'écriture, telle qu'on l'a connue, c'est-à-dire avec très peu d'aide machinique, ce que la photographie fut aux métiers de la mimésis qui se servaient uniquement du système nerveux et de la main. Je ne pense pas que cette écriture éliminera l'écriture classique. Pas à l'état actuel, en tout cas. Je pense plutôt que, tout en se développant dans sa propre direction, elle forcera les écrivains vers de nouveaux domaines exploratoires. Et ces espaces nous intéressent parce qu'ils seront proprement humains. Les autres sont simulables. En effet, si la machine peut simuler un énoncé humain assez complexe et plein de significations diverses, cela va élaborer dans la sensibilité à la langue des domaines non simulables à l'informatique. Mais à l'inverse, l'informatique, de la même façon que la photo, en tant que produit scientifique et technique, va engendrer ses propres sensibilités discursives. Là également on peut s'attendre à du nouveau. 
Le générateur informatique partage avec la photographie la reproductibilité, le niveau multiple d'écriture, la simulation et l'indifférence : 1) chaque photo est en effet un double et non un original. Elle est, par la nature de la technique, indéfiniment reproductible; de même, le médium informatique est essentiellement reproductible; 2) la photo est le produit d'une technique chimique et optique assistée de mécanique qui n'a jamais cessé de varier et d'augmenter avec l'évolution de son concept, de même Prisme est écrit dans le langage Turbo Prolog, mais varie selon le caprice des utilisateurs; 3 ) la fonction physique simulée par la photo est la relation cristallin/rétine (focaliser/enregistrer) et le dessin, alors qu'avec Prisme, c'est la fonction écriture/lecture; 4) enfin, la photo voit du même oeil le meurtre sadique et le sourire de l'enfant, alors que le générateur a la même indifférence à l'égard de toutes les significations qu'il agence. La photo enregistre tournée vers le réel, le générateur syntaxique aléatoire enregistre tourné vers la langue et l'océan du sens.

La langue froissée sur elle-même, la plage avec ses galets, l'appareil de photo sont des métaphores qui portent l'esprit vers des aspects de ce qui s'offrent ici : de nouveaux rivages. Ça donne à lire, ça donne à rire.

La machine introduit le disparate au coeur de chaque énoncé. Le poète moderne pourrait y entendre des résonances, y chercher lui aussi des vers bien faits, des collusions prégnantes entre mots. On fait de la poésie avec des mots et pas avec des idées, aurait dit Mallarmé, et c'est exactement ce que fait Prisme, au sens propre. Il conjoint au hasard des mots, il disjoint le sens. II donne à penser, un peu comme les symboles à la manière kantienne, mais avec une connotation très matérielle d'indifférence. Le symbole donne à penser une suite ininterrompue et indéterminée d'interprétations dont l'esprit est le lieu.

Un dramaturge, par exemple, pourra simuler une conversation entre plusieurs personnages. Car, en fin de compte, un personnage n'est qu'un lexique et la récurrence de certains types de phrases, longues ou courtes, ponctuées d'interjections ou non. On se retrouve alors dans un salon complètement 
dément où chacun parle pour soi avec la plus grande politesse.

La théorie des jeux et de la stratégie stipule que moins un coup est prévisible, plus fort il est. On peut supposer qu'un échange de propos dans des lieux décisionnels requiert certains énoncés qui ont pour but de désarçonner l'interlocuteur. II semble qu'un générateur d'énoncés aléatoires est une excellente source d'énoncés qui viennent de nulle part, mais qui, en même temps, sont bien là. Si quelqu'un les prononce, les donne à entendre comme faisant partie de la configuration dans laquelle il est inséré, ils résonneront avec le contexte.

Je veux parler, enfin, de deux autres usages : l'un gymnastique et l'autre heuristique. Supposons quelqu'un qui décide d'en faire une gymnastique, comme cela est tellement à la mode actuellement, mais, ici, mentale. II choisit avec attention une certaine variété de vocabulaire et de structure qu'il explore ensuite. Mettons qu'il lise une centaine d'énoncés à tous les matins, sur un thème arbitraire? Son plaisir consiste à saisir où passe la différence entre le banal, l'exceptionnel, le confus, l'oxymoron, la contradiction, l'absurde, la poésie de l'image, la théorie, le conseil technique, la monstrueuse grivoiserie ou le désintérêt.

La méthode du "brain storming" existe déjà depuis plusieurs décennies. Elle tirait déjà sa force de la recherche du nouveau, par des sources indépendantes d'idées grâce à la réunion de plusieurs individus nécessaire à l'obtention d'une bonne variété. II est relativement facile actuellement de recueillir le vocabulaire d'un domaine technique et celui de son contexte, et de l'agencer à des structures syntaxiques connues dans ce domaine, incluant les prénoms des chercheurs par exemple. Dans ce sens, la machine informatique est une aide comme orageur de cerveau, une heuristique aléatoire.

Cette façon de traiter la langue est à l'écriture ce que la photographie est au dessin. Elle ne peut tuer la créativité parce qu'elle n'en est qu'un moyen. Personne n'y est obligé.

Elle est superficielle, ennuyeuse, drôle, sérieuse, et prodigue. Je donne, tels quels, quelques exemples seulement, ne 
pouvant évidemment pas en présenter une centaine de pages, ce qui serait, par ailleurs, la bonne façon. Le vocabulaire comprend le dictionnaire des symboles, le dictionnaire philosophique de Lalande, quelques expressions usuelles de l'esthétique et quelques structures syntaxiques simples qu'on reconnaîtra au passage.

Nudité qui construit ne déploie pas relativités!

Des immoralistes esthétiques aéraient le calendrier idéologique. II n'y a pas de minimum sans moralistes!

Qui boit un équivalent boit des disques.

Un incandescent peuplé aura embelli du sagittaire tressé.

Transmigration qui cristallise ne saute pas manie!

Les identificatrices eurent certifié les attitudes chevalines.

II n'y a pas de mystiques sans sablier!

Comme les cheveux sont quelconques!

II n'y a pas de chariot sans despote!

Les faucilles aèrent le simple.

Qui tourbillonne sonne!

Je suis une boiteuse qui respire!

Bibliothèque qui jaunit n'illumine pas axiomatique!

Les exemples qui suivent ont un plus grand degré de hasard

De la sage haute cavalée les pèlerins nus.

Les maçons cuirassés provoqueraient les Sphinx.

Les Égyptes incluses avoir doublé les Pléïades tactiles.

Les autres prostituées lieraient des Hobbes culbutés.

Les Lord Bertrand Russell royaux les visionnaires kakis.

Des polémiques ruinées tonneraient du marteau ouvert.

De la menteuse emmurée ait naturalisé de la sage choisie. 
Ici des structures simples

Je suis une cabale qui roussit!

Je suis un roseau qui marche!

Tamarinier exact!

Pourquoi les anarchistes cuvent-ils les auréoles? Parce qu'ils greffent l'hyperréaliste!

Je suis une tempérance qui écrit!

Qui invente des loyalismes invente un airain.

Mythes, vous mordrez moyennement!

Un tohu-bohu de frocs jamais ne spécifiera le froment.

Si seulement la colonne était une jambe!

Antinomie qui gouverne ne sale pas dualité!

Pourquoi les somnambules uniformisent-ils le minuit?

Si seulement la terminologie était un sort!

Les bâtons vampirisés copiaient la frange.

Savez-vous qui a simulé la limite? Simone de Beauvoir.

Vous classifiiez immoralement les fanatiques!

Savez-vous qui a regardé l'échange? Whitehead.

Savez-vous qui a incubé les éléments? Pollock.

Savez-vous qui a inquiété les blés? Diane la chasseresse.

Savez-vous qui a exposé le sexe? Voltaire.

Savez-vous qui a sauvé le respect? Hannah Arendt.

Le savant, alors l'apocalypse, chante. 
Pour finir voici quelques petits poèmes en style indirect libre, suivis par des exemples de ce qui se passe quand la fonction «néologisme» est activée au maximum.

Des chiendents, des caricaturistes, quatorze imaginations dénaturent, peupler. Avoir enchaîné des politiques.

Des papiers, un amour, dix nectars fortunent.

Souvenir des coutumes.

Des éternités, des unijambistes, soixante ascèses entrelacent, boire. Avoir cloîtré une boucle.

Des affirmations, des despotes, onze titres coffrent, avoir. Avoir neigé des chats.

Des institutions, un cyclope, mille appétits ramifient, paraître. Avoir pêché des contraintes. 
Des tarots,

des ricins,

deux lumières versent,

rêver. Doubler un lézard.

Les météofuges pseudopsychisent un hétéronome multitemporel. lls l'ont dit dans un gros livre!

Vous aviez cacomancisé implicitement la démalepsie!

Un héliocide halokinésique viviurge des ortholèxes

xénothérapiques.

Comme le métrogène est talamotechnique!

La décadimensionnalité avait viviandrisé les tératomotricités élatérodocimasiques.

\section{Appendice}

Prisme est un objet informatique composé par Claude Frascadore et l'auteur de cet article, depuis octobre 1985. Au début on le nommait Générateur Syntaxique. Assez tôt, il fut écrit, pour un ordinateur personnel, dans un langage de $5^{\circ}$ génération nommé Prolog, oeuvre de Alain Colmerauer au début des années 70, à Marseilles, dans le but de servir aux superordinateurs de recherche en intelligence artificielle et en systèmes experts. C'est ce langage, transformé pour des ordinateurs personnels sous le nom de Turbo Prolog, que nous avons utilisé. Prolog est une abréviation de programmation logique. C'est un langage déclaratif et non procédural, c'est-àdire que l'usager définit des faits et des lois que le programme compile ensuite pour former le logiciel utilisable. Prisme en est à sa troisième version qui date de 1989. II est écrit en Turbo Prolog, version 2.0, et a comme dimension 3,5 méga-octets. 


\section{Bibliographie}

Frascadore, Claude et François Raymond. "Le Père Noël enfantin", Le Devoir. Montréal (23 décembre 1983), p. D1, suivi d'un court article de Guy Ferland, p. D2.

Ferland, Guy. "Un logiciel pas comme les autres", Le Devoir. Montréal (24 février 1990).

Frascadore, Claude et François Raymond. "Les ordinateurs seront-ils les nouveaux artistes?", Interview par Pierre Lapalme dans le cadre de l'émission Tangentes, RadioCanada (17 décembre 1992).

François Raymond Collège Édouard-Montpetit 